\title{
Design and Implementation of a Single Layer Circularly Polarized Reflectarray Antenna with Linearly Polarized Feed
}

\author{
F. Ahmadi, Student Member IEEE, A. Namiranian, and B. Virdee, Member, IEEE
}

\begin{abstract}
A novel microstrip unit-cell element with a simple structure is presented for designing circularly polarized reflectarray that converts linearly polarized incident field into a radiated circularly polarized field. The unit-cell element consists of a circular patch with two orthogonal slots etched in it. A parametric study was carried out using CST microwave studio on the unit-cell element to evaluate the effect of the unitcell's salient parameters. This unit-cell element was used to design and implement a prime-focus array, which had 1-dB gain bandwidth and 1-dB axial-ratio bandwidth of $\sim 5 \%$.
\end{abstract}

Index Terms-Circularly polarized, reflectarray antenna, linearly polarized.

\section{INTRODUCTION}

$\mathrm{C}$ OMBINED with some of the best features of reflector antennas and phased array antennas, the reflectarray antenna is considered to be an attractive replacement for applications that require a low profile antenna with a high gain performance. Essentially reflectarray antennas comprise of a planar array of radiating elements illuminated by a feed source. The phase of the reflected wave at each unit-cell element is carefully controlled to create a planar phase front in its far-field region. Since the introduction of microwave integrated technology (MIC), the microstrip patch has become very popular, and has inspired interest in the development of planar reflectarray antennas [1]. Compared to array antennas, the reflectarray eliminates the complexity and losses of the feeding network.

Circular polarization (CP) is employed in numerous applications, such as in satellite communication, because of its insensitivity to transmitter and receiver orientation and it's robustness against environmental interface. Circular polarization antenna is used as a feed in CP reflectarrays. Example of this type of feed is found in [2] where the reflectarray antenna includes a unit-cell element comprising of a rectangular patch and a rectangular ring. In another example the $\mathrm{CP}$ feed illuminates patches with different rotation angles [3]. CP reflectarrays can also be designed using a linearly polarized feed. In this case the elements are designed so that the $\mathrm{CP}$ incident field is decomposed into two equal components with a necessary phase shift to radiate a CP field [4].

${ }^{1} \mathrm{~F}$. Ahmadi is with Electrical Engineering Department,Tarbiat Modares University, Tehran, Iran. (e-mail: f.ahmadi@modares.ac.ir).

${ }^{2} \mathrm{~B}$. Virdee is with Center for Communications Technology, Faculty of Life Sciences and Computing, London Metropolitan University, London, UK.
In this paper we have designed a simple and small-sized unit-cell element that can be employed for circular polarization with a linearly polarized feed. This unit-cell element is composed of circular patch that is loaded with two orthogonal elliptical slots. The element was etched on single layer of FR4 substrate, and the reflectarray comprising of this type of element is easy and cheap to fabricate compared with the previous works [5]-[8]. The performance of this unit-cell element was investigated by designing 144-element array. The measurement results of the array show a $1-\mathrm{dB}$ gain bandwidth and $1-\mathrm{dB}$ axial-ratio bandwidth of $\sim 5 \%$. These results are much better compared with [5]-[7]. In fact the proposed reflectarray has a better axial-ratio bandwidth compared with [9]-[12], a better sidelobe level (SLL) in comparison with [13], and a better radiation efficiency in comparison with [8].

\section{ANALYSIS OF THE PROPOSED UNIT-CELL}

The geometry of the proposed reflectarray unit-cell element is shown in Fig.1. This element is implemented on a single layer circular microstrip patch that is loaded with two orthogonal intersecting elliptical slots. A conducting ground-plane is placed on the other side of the substrate. The required phase shift at each position on the reflectarray surface is obtained by adjusting the length of the elliptical slots. The dimensions of the unit-cell element are chosen to resonate at $9.4 \mathrm{GHz}$.

The electric field of the excitation wave is polarized by the two slots to create two orthogonal waves each of which is along the length of the slot, and re-radiated. A CP wave is reflected if 90 degree phase difference exists between these two waves. Hence the lengths of the two slots need to be designed to have an electrical difference of 90 degrees in order to produce a 90 degree phase shift between the two reflected waves.

Various values for unit-cell parameters were investigated in the simulation analysis in order to obtain optimum performance of the linear phase curves and achieve the greatest range of phase shift. The optimized values of the all parameters are defined in Table I. The simulation was conducted using CST microwave studio.

The simulated phase curves versus the length of the slot line are shown in Fig. 2 for two polarizations at the operating frequency. The phase of the two polarizations is equal at a slot length of approximately $7.3 \mathrm{~mm}$. In Fig. 3 phase variation are shown at three different frequencies, i.e. 9.2 GHz, 9.4 GHz and 9.6 GHz, for the two polarizations. As can be seen, phase curves are approximately linear across a slot of length between $5-7 \mathrm{~mm}$, and the overall 
phase range is about 325 degrees.

Fig. 4 shows the phase variation as a function of slot length at the operating frequency of $9.4 \mathrm{GHz}$ for different angles of wave incidence to the unit-cell. Analysis shows for incident angles less than 20 degrees provide the smallest difference between the phase curves as a function of slot length. In fact the variation was small when the slot length was between approximately 5.5 and $7.5 \mathrm{~mm}$, as shown in Fig. 4 .

\section{ANALYSIS OF THE ARRAY}

In order to validate the performance of the elements a 144-element reflectarray was designed at an operating frequency of $9.4 \mathrm{GHz}$. The fabricated $24 \times 24 \mathrm{~cm}^{2}$ center-fed antenna is shown in Fig. 5. The antenna is fed with a linearly polarized pyramidal horn antenna, with an aperture size of $3.1 \times 5.4 \mathrm{~cm}^{2}$. The feed is rotated 45 degrees relative to the aperture axis in order to excite two orthogonal slots in each cell. The distance between the feed and the center of the reflectarray antenna is $29.5 \mathrm{~cm}$. The $F / D$ ratio was chosen to be 1.23 in order to achieve the best trade-off between the spillover and illumination efficiency.

The feed is placed far enough from the reflectarray so that the incident field can be approximated by a plane wave. The basic design principle requires that the phase of the reflected field from an element in the reflectarray be chosen so that the total phase delay in front of the reflectarray is constant for all elements. The required phase shift can be obtained by this the expression:

$$
K_{0}\left(R_{i}-\vec{r}_{i} \cdot \hat{r}_{0}\right)-\psi_{i}=2 \pi N
$$

Where $K_{o}$ is the wave number, $R_{i}$ is the phase center of the feed to the $i$-th element, $\vec{r}_{i}$ is the position vector from the center of array to the $i$-th element, and $\hat{r}_{o}$ is unit vector along the main beam direction. The required length of the crossslots in each element was obtained by using equation (1) and Fig. 2.

Simulated RHCP and LHCP radiation patterns of the reflectarray at $9.4 \mathrm{GHz}$ are shown in Fig. 6. CST microwave studio is used to calculate far-field patterns. The measured and simulated radiation far-field patterns are compared in Fig. 7. As can be seen from these figures, a peak gain of $21.9 \mathrm{~dB}$ is obtained at $9.4 \mathrm{GHz}$ where the cross polarization level is $18 \mathrm{~dB}$ lower than the main beam peak and SLL is $18 \mathrm{~dB}$. The measured antenna efficiency at $9.4 \mathrm{GHz}$ was $21.8 \%$. The antenna's performance is better in terms of efficiency in comparison with [8], and the SLL is better compared to [13]. As shown in [3], by using offset feed eliminates aperture blockage and provides greater radiation efficiency. The gain and axial-ratio are shown in Fig. 8 and Fig. 9, respectively. From these figures it can be observed that both of the $1-\mathrm{dB}$ gain bandwidth and 1-dB axial-ratio bandwidth are $5 \%$.

\section{CONCLUSION}

The feasibility of a novel single-layer circularly polarized reflectarray was practically demonstrated. The required phase shift is obtained by varying the length of the two orthogonal elliptical slots embedded in the circular patch. There is very good correlation between the simulated and measured far-field radiation patterns. Compared to previous work the antenna provides a good performance in terms of radiation efficiency, side-lobe level, gain bandwidth, and axial-ratio bandwidth.

\section{REFERENCES}

[1] D.G. Berry, R.G. Malech, and W.A. Kennedy, "The reflectarray antenna,” IEEE Trans. Antennas Propagat., Vol. 11 , Nov. 1963 , pp. $645-651$.

[2] Q.Y. Li, Y.C. Jiao, and G. Zhao, "A novel microstrip rectangular patch ring combination reflectarray element and its application," IEEE Antennas Wireless Propag., Letter, Vol. 8, pp. 1119-1122, 2009.

[3] J. Huang and R. J. Pogorzelski, "A Ka-band microstrip reflectarray with elements having variable rotation angles," IEEE Trans. Antennas Propag, Vol. 46, No. 5, pp. 650-656, May 1998.

[4] G. Zhao, Y-C. Jiao, F. Zhang, and F-S. Z, "A subwavelength element for broadband circularly polarized reflectarrays," IEEE AWPL, Vol. 9, 2010, pp. 330-333.

[5] M.R. Chaharmir, J. Shaker, M. Cuhaci, A. Sebak, "Circularly polarised reflectarray with cross-slot of varying arms on ground plane," Electronics Letters, 2002, pp. 1492 - 1493.

[6] M.R. Chaharmir, J. Shaker, M. Cuhaci, "Development of a dual band circularly polarized microstrip reflectarray," 33rd European Microwave Conference, 2003, pp. 1075 - 1078.

[7] M. Albooyeh, N. Komjani, and S.M. Mahani, "A circularly polarized element for reflectarray antennas," IEEE Antennas and Wireless Propagation Letters, vol. 8, 2009, pp. 319-322.

[8] S.H. Zainud-Deen, Abd-Elhady, A.A. Mitkees, A.A. Kishk, "Design of dielectric resonator reflectarray using full-wave analysis," National Radio Science Conference, NRSC, 2009, pp. 1-9.

[9] J. Haung, J.R. Pogorzelski, "A Ka-band microstrip reflectarray with elements having variable rotation angles," IEEE Trans. on Antennas and Propagation, Vol. 46, No. 5, 1998, pp. 650-656.

[10] D.M. Pozar, S.D. Targonski, and D. Syrigos, "Design of millimeter wave microstrip reflectarray," IEEE Trans. on Antennas and Propagation Letters, Vol. 45, 1997, pp. 287-296.

[11] H. Chulmin, K. Chang, "Ka-band reflectarray using ring elements," Electronics Letters, 2003, pp. $491-493$.

[12] A. Yu, F. Yang, A.Z. Elsherbeni, J. Huang, Y. Kim, "An offset-fed Xband reflectarray antenna using a modified element rotation technique," IEEE Trans. on Antennas and Propagation, 2012, pp. $1619-1624$.

[13] S. Adel, H.A. Hammad, "A novel microstrip rotating cell for CPreflectarray applications," IEEE Radio and Wireless Symposium, 2003, pp. 121-123.

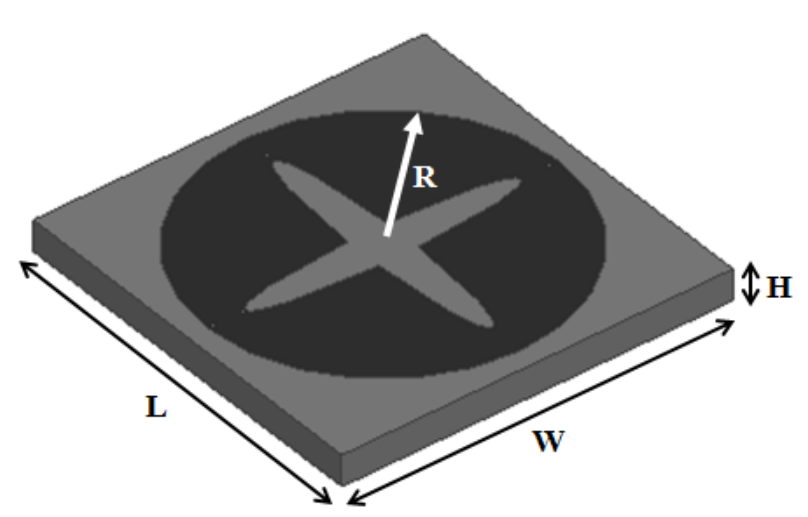

Fig. 1. Proposed unit-cell.

Table I. Dimensions of the proposed unit-cell

\begin{tabular}{|c|c|c|c|c|}
\hline \multicolumn{5}{|c|}{ Substrate } \\
\hline \multirow{2}{*}{ FR4 } & $\varepsilon_{r}$ & Thickness (mil) & W $(\mathrm{mm})$ & $\mathrm{L}(\mathrm{mm})$ \\
\cline { 2 - 5 } & 4.4 & 31 & 20 & 20 \\
\hline \multicolumn{4}{|c|}{ Microstrip patch } \\
\hline $\mathrm{R}(\mathrm{mm})$ & 9 & Slot dimension \\
\hline \multicolumn{4}{|c|}{ Axial-ratio } \\
\hline \multicolumn{2}{|c|}{$1 / 7$} \\
\hline Large axis & \multicolumn{3}{c}{} \\
\hline Variable & \multicolumn{3}{c|}{} \\
\hline
\end{tabular}




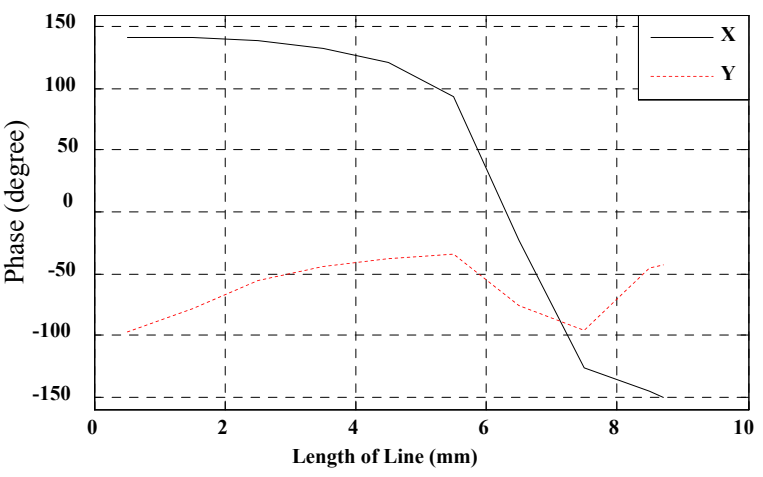

(a)

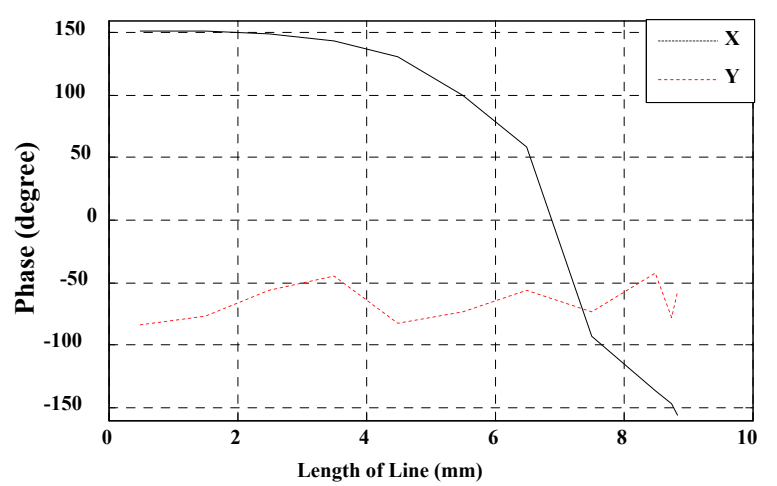

(b)

Fig. 2. Phase curves versus slot length: (a) X-polarized incident wave, and (b) Y-polarized incident wave.

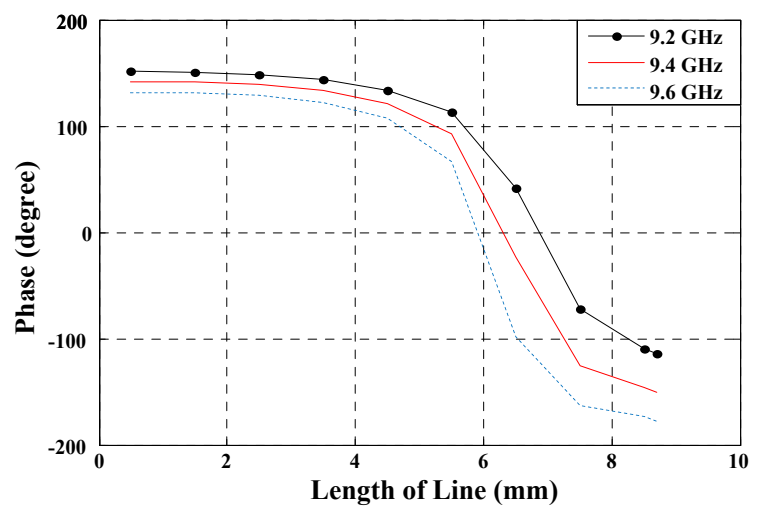

(a)

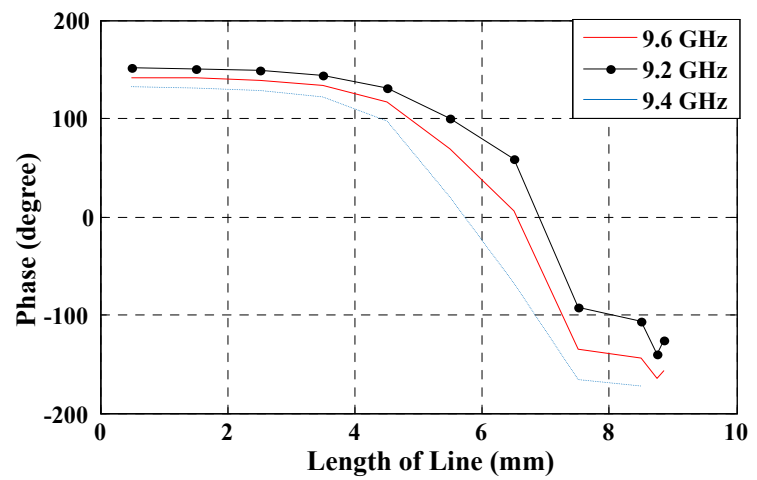

(b)

Fig. 3. Phase curves at three different frequency: (a) X-polarized incident wave, and (b) Y-polarized incident wave.

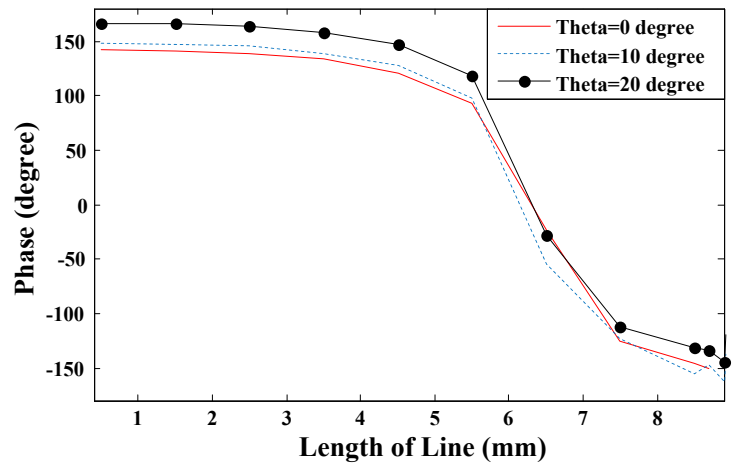

(a)

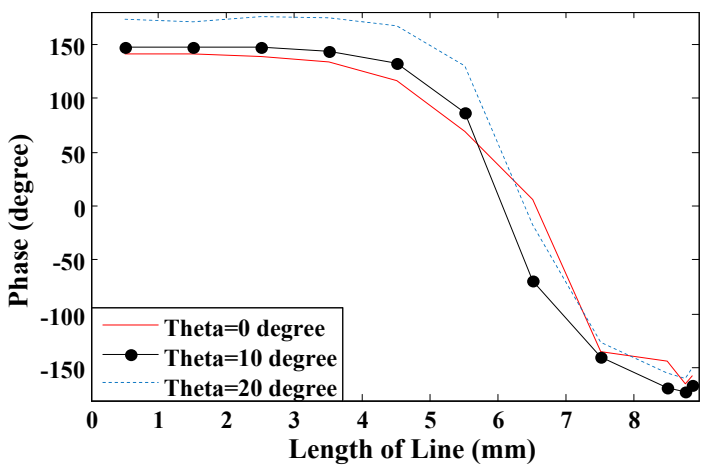

(b)

Fig. 4. Simulated phase of the reflection coefficient versus the arm length of the slot at different incident angles with $\varphi=0^{\circ}$ : (a) Xpolarized incident wave, and (b) Y-polarized incident wave.

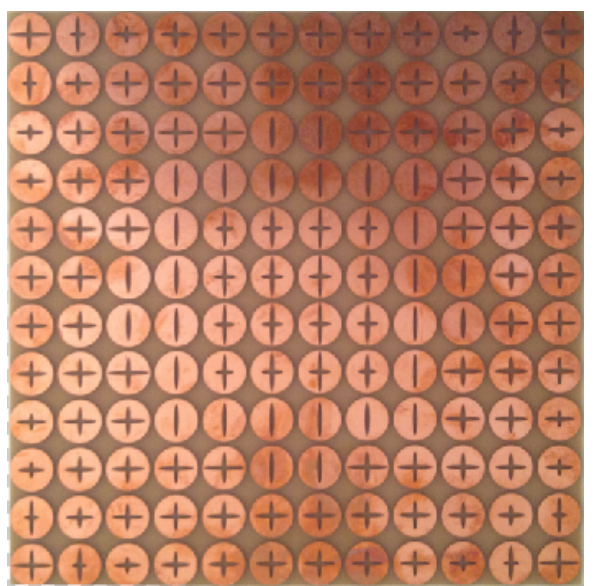

Fig. 5. Fabricated reflectarray structure.

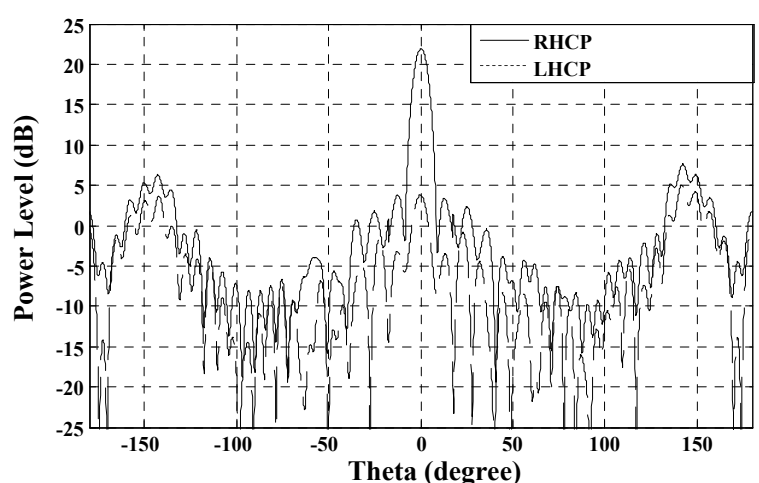

(a) 


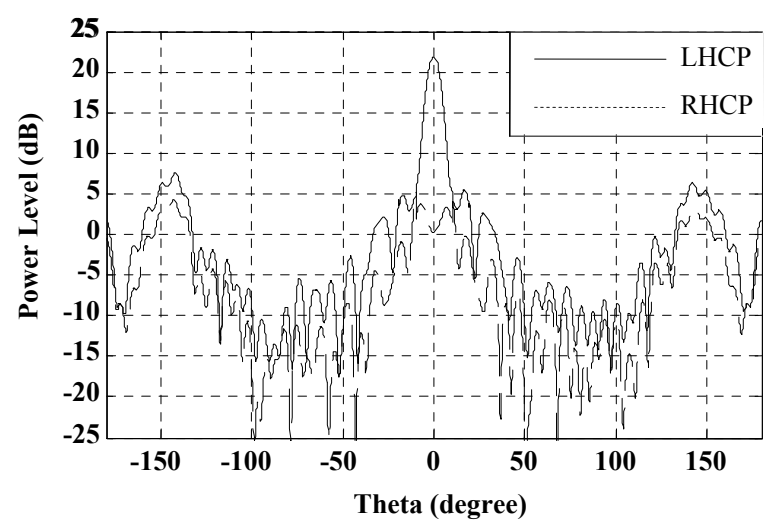

(b)

Fig. 6. CP polarized farfield pattern at $9.4 \mathrm{GHz}$ : (a) $\varphi=0^{\circ}$, and (b) $\varphi=90^{\circ}$.

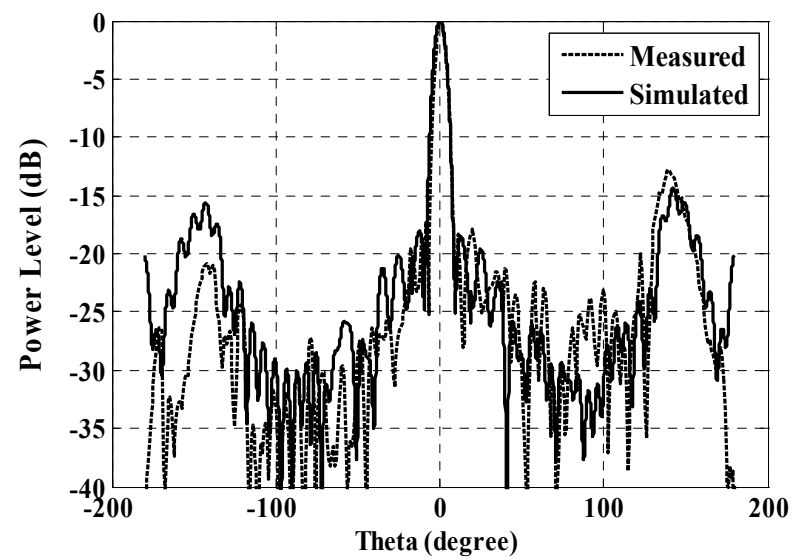

Fig. 7. Measured and simulated far-field pattern at $9.4 \mathrm{GHz}$.

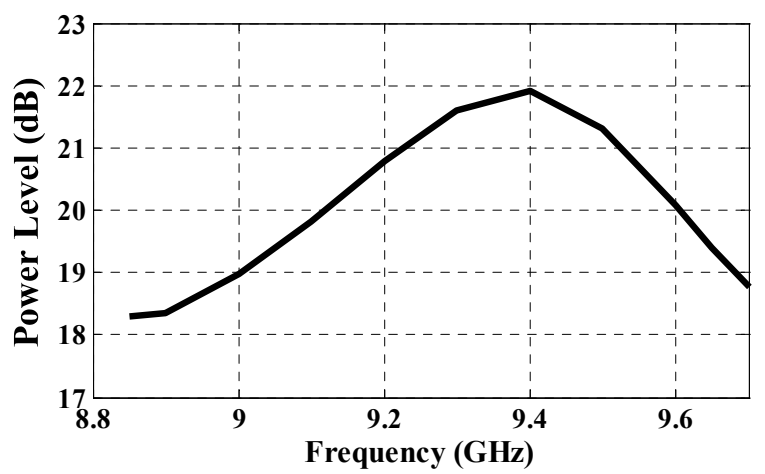

Fig. 8. Gain versus frequency.

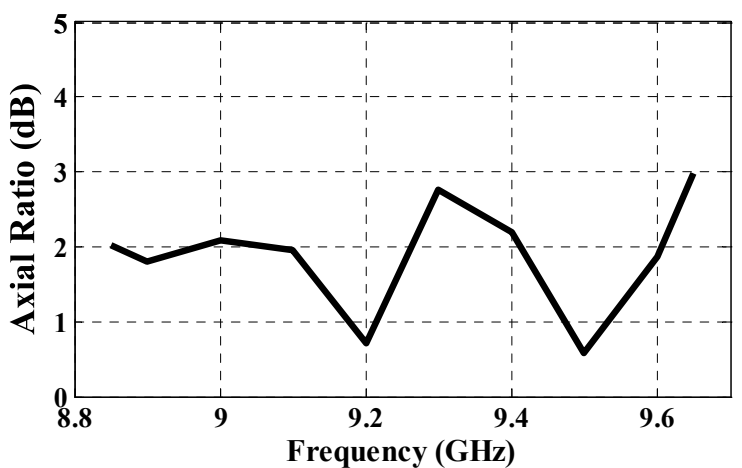

Fig. 9. Simulated axial-ratio versus frequency. 